\title{
Risk scoring system for recurrence after simultaneous resection of colorectal cancer liver metastasis
}

\author{
Yuchen $\mathrm{Wu}^{1,2 \#}$, Tianan Guo ${ }^{1,2 \#}$, Zhenhong $\mathrm{Xu}^{1,2 \#}$, Fangqi Liu ${ }^{1,2}$, Sanjun Cai ${ }^{1,2}$, Lu Wang ${ }^{2,3}$, Ye $\mathrm{Xu}^{1,2}$ \\ ${ }^{1}$ Department of Colorectal Surgery, Fudan University Shanghai Cancer Center, Shanghai, China; ${ }^{2}$ Department of Oncology, Shanghai Medical \\ College, Fudan University, Shanghai, China; ${ }^{3}$ Department of Hepatic Surgery, Fudan University Shanghai Cancer Center, Shanghai, China \\ Contributions: (I) Conception and design: Y Wu, S Cai, L Wang, Y Xu; (II) Administrative support: S Cai, L Wang, Y Xu; (III) Provision of study \\ materials or patients: Y Wu, T Guo, Z Xu, F Liu; (IV) Collection and assembly of data: Y Wu, T Guo, Z Xu; (V) Data analysis and interpretation: Y \\ Wu, T Guo; (VI) Manuscript writing: All authors; (VII) Final approval of manuscript: All authors. \\ "These authors contributed equally to this work. \\ Correspondence to: Ye Xu. Department of Colorectal Surgery, Fudan University Shanghai Cancer Center, Shanghai 200032, China; Department \\ of Oncology, Shanghai Medical College, Fudan University, 270 Dong An Road, Shanghai 200032, China. Email: yexu@shmu.edu.cn; Lu Wang. \\ Department of Hepatic Surgery, Fudan University Shanghai Cancer Center, Shanghai 200032, China; Department of Oncology, Shanghai Medical \\ College, Fudan University, 270 Dong An Road, Shanghai 200032, China. Email: wang.lu99@hotmail.com; Sanjun Cai. Department of Colorectal \\ Surgery, Fudan University Shanghai Cancer Center, Shanghai 200032, China; Department of Oncology, Shanghai Medical College, Fudan \\ University, 270 Dong An Road, Shanghai 200032, China. Email: caisanjun_sh@163.com.
}

Background: The simultaneous resection of synchronous colorectal cancer liver metastasis (SCRLM) has been widely applied. It is necessary to establish a risk scoring system to predict post-operative recurrence, especially in patients with neoadjuvant treatment.

Methods: The medical records of 221 patients undergoing simultaneous resection of CRLM were assessed in this study with a further 128 patients allocated to a validation group. All patients in the study group were classified according to their history of neoadjuvant treatment and univariate and multivariate analyses were applied to study independent risk factors. A score model was then generated according to the factors included. Our data set were also applied to validate three other existing scoring models [Fong clinical recurrence score (CRS), Konopke, and Zakaria disease-free survival (DFS) score], and the concordance index was calculated for comparison among these models.

Results: CRLM involving more than three nodes positive for a primary tumor was considered an independent risk factor for progression in patients without neoadjuvant treatment and all score models could discretely stratify patients according to disease free survival. In patients receiving neoadjuvant treatment, CRLM involving more than one node and transfusion invasion were major determinants in patients after treatment. However, only our scoring system and Fong's CRS score could discretely discriminate patients. In the validation group, patients were significantly classified with the score system.

Conclusions: Existing score models had better values for determining prognosis in patients with SCRLM, especially in those undertaking neoadjuvant treatment. Larger cohorts, along with more detailed clinical features and multicenter validation should be undertaken before utilization.

Keywords: Colorectal cancer liver metastasis; simultaneous resection; neoadjuvant treatment; risk scoring model

Submitted Mar 22, 2021. Accepted for publication Jun 11, 2021.

doi: $10.21037 /$ atm-21-2595

View this article at: https://dx.doi.org/10.21037/atm-21-2595 


\section{Introduction}

Colorectal cancer (CRC) is one of the most common cancers worldwide (1) and the common cause of cancerrelated death in China (2). Colorectal liver metastasis (CRLM) is the most common metastasis and cause of mortality $(3,4)$ and it is estimated that $25 \%$ of CRC patients have synchronous colorectal cancer liver metastasis (SCRLM).

Radical surgery is the only curative treatment for patients with CRLM and considering the surgical difficulties and perioperative complications involved, a usual strategy for synchronous CRLM is to perform two-stage resection which sees primary and metastasis resection in different periods. Chemotherapy can also be applied in the interval to down-stage metastasis and eradicate micro-metastasis (5), and a liver-first management is also applied in many centers (6). However, because of improvements in surgical technology and postoperative care, simultaneous resection has been increasingly performed, based on the proof that postoperative mortality, morbidity, and even longterm outcome were comparable to that achieved using staged resection (7-11), let alone the benefits obtained from less hospital stay and expense $(12,13)$. On this basis, simultaneous resection for SCRLM has been widely accepted.

Neoadjuvant treatment has also been applied to increasing numbers of patients with SCRLM before surgery to increase the resectability rate and to monitor the response (5). We have previously shown that neoadjuvant treatment did not independently increase postoperative complications (14), although the prognosis of patients receiving this treatment requires further study.

There have been several studies and score systems focusing on the long-term prognosis of CRLM, and most were confounded with multiple factors, such as synchronous and metachronous, simultaneous and two-stage resection, or with and without neoadjuvant treatment. On SCRLM, Lee has established a scoring system of overall survival in patients with simultaneous. Considering the small sample size and no validation group, further research is required (15).

The investigation of the increasing use of simultaneous resections, especially after applying neoadjuvant treatment, requires a stratification study, and herein, we induced the SCRLM database in our center from 2015 to 2018, to study the risk factors for recurrence in patients after simultaneous resection, with or without neoadjuvant treatment. In addition, several existing score systems were introduced in our data and further compared. We present the following article in accordance with the TRIPOD reporting checklist (available at https://dx.doi.org/10.21037/atm-21-2595).

\section{Methods}

\section{Patient selection and medical management}

This single-center, retrospective study reviewed a database of patients treated in the Department of Colorectal Surgery at the Shanghai Cancer Center, from June 2015 to March 2018. Patients who met the following criteria were selected: (I) initially diagnosed as having synchronous CRLM; (II) Eastern Cooperative Oncology Group status $\leq 2$, indicating no surgical contradictions and with simultaneous resection of primary tumor and liver metastasis; (III) pathologically confirmed malignant primary tumor and liver metastasis; (IV) available and complete clinical records, including pathologic diagnosis, treatment strategy, and follow-up. The exclusion criteria included patients who had either malignant tumors in other organs, or palliative resection of tumors. The RAS (KRAS and NRAS) and BRAF mutations were detected in 144 patients, although not all patients chose to have this investigated.

Of the 229 patients included, neoadjuvant therapies were applied to 98 patients and the chemotherapy regimen was Oxaliplatin based $(\mathrm{N}=82)$, including XELOX and mFOLFOX6; a FOLFIRI regimen $(\mathrm{N}=13)$; and three patients received both because of the progression of liver metastasis. The number of chemotherapy cycles ranged from three to ten. In patients with low and cT3 rectal cancer, 17 also received long-term neoadjuvant radiotherapy and 30 patients were additionally treated with Bevacizumab $(\mathrm{N}=20)$ and Cetuximab $(\mathrm{N}=10)$. All patients underwent radical resection at least 30 days after neoadjuvant therapy was completed and adjuvant chemotherapy was applied to all patients after simultaneous surgery, according to regiments before surgery. Simultaneous surgical resections were performed by surgeons from both the departments of colorectal surgery and hepatobiliary surgery, and intra operative ultrasound (IOUs) was performed to confirm the number and location of liver metastasis.

A further 128 patients presenting between April 2018 to December 2018 were included in validation group and all patients in the study received strict follow-up every 3 months up to the time of writing. The detailed clinical characteristics of patients are listed in Table S1.

Blood CEA levels and CT/MRI imaging of the chest, abdomen, and pelvis were performed to evaluate progression. The interval between simultaneous resection 
and recurrence, termed as disease-free survival (DFS), was calculated once any confirmed evidence of metastasis occurred. The study was conducted in accordance with the Declaration of Helsinki (as revised in 2013), and was approved by the Medical Ethics Committee of Fudan University Shanghai Cancer Center. Written informed consent was obtained from all participants.

\section{Statistical analysis}

Statistical analysis was performed using STATA version 15 and $R$ version 3.5.1 and a $P$ value of $<0.05$ was considered as significant in all analyses.

Chi-square tests and Fisher's exact tests were used to compare categorical variables between the groups (with or without neoadjuvant treatment) and the KolmogorovSmirnov test was used to verify the normal distribution of variables. The exploratory comparison of normally distributed and non-normally distributed independent groups was performed using $t$-tests and Mann-Whitney $\mathrm{U}$ tests (two groups).

For survival analysis, curves were plotted using the Kaplan-Meier method and analyzed by the logrank test. Univariate and multivariate analyses to identify prognostic predictors were performed using Cox proportional hazard models and backwards elimination was applied. Variables were included if $\mathrm{P}$-values were under 0.05, while removed if over 0.10 . Patients were then stratified into risk groups according to the significant factors in multivariate analysis. Another three models [Fong clinical recurrence score (CRS) (Points: 0-2 vs. 3-5) (16), Konopke score (Points: 0 vs. 1 vs. 2 to 3) (17) and Zakaria score (Points: 0 vs. 1 to $3 v s .4$ or hepatoduodenal node positive) (18)] were assessed to predict the recurrence of liver metastasis. Fong developed a prognostic scoring system based on five parameters; the largest CRLM size more than $5 \mathrm{~cm}$; CEA levels more than 200; primary lymph nodes (N stage) positive; liver metastasis more than one; and interval between primary resection and recurrence of CRLM less than 4 months. The Konopke score includes three factors: liver metastasis more than three, CEA levels more than 200, and SCRLM. Finally, the Zakaria system includes four risk factors; primary lymph nodes ( $\mathrm{N}$ stage) positive; liver metastasis more than 1; transfusion; and hepatoduodenal node positive.

The predictive accuracy of each model was evaluated by calculating the concordance index (C-index) in patients with follow-up of at least 2 years. A C-index close to 0.5 indicated that the scoring system is no better than chance at predicting patient survival, while a score of 1 indicates perfect prediction. Ninety-five percent confidence intervals (95\% CIs) were calculated using bootstrap resampling with 1000 replications.

\section{Results}

\section{Comparison of clinical characteristics between patients with or without neoadjuvant treatment}

Altogether 229 patients with simultaneous resection of CRLM were included, in which $98(42.8 \%)$ also received neoadjuvant treatment, and a comparison of clinical characteristics is listed in Table 1. The results show that sex, BMI, site of primary tumor, max diameters of liver metastasis, CEA level, transfusion, complications, differentiation, perineural invasion RAS, and BRAF mutation rate were almost balanced between the two groups. However, compared to those not receiving neoadjuvant treatment, those that did were younger $(\mathrm{P}=0.046)$, had more liver metastasis $(\mathrm{P}=0.017)$, and were mostly bilobar in distribution $(\mathrm{P}=0.030)$. T stage $(\mathrm{P}=0.002)$ and $\mathrm{N}$ stage $(\mathrm{P}=0.006)$ were also comparably lower in those not receiving neoadjuvant treatments, and lymphovascular invasion and mucinous pathology types were more common in patients without pre-operative treatment $(\mathrm{P}=0.043$ and $\mathrm{P}=0.025$, respectively).

\section{Prognostic factors analysis}

We used X-tile Software (http://medicine.yale.edu/lab/ $\mathrm{rimm} / \mathrm{research} /$ ) to determine the cutoff values of CRLM numbers, based on the minimum $\mathrm{P}$ values from the log-rank chi-square statistics (Figure S1). This resulted in a cut-off in three metastases being chosen for the non-treatment group, while one was chosen for the other group.

Altogether 122 patients had cancer progress. Univariate analysis revealed that the number of hepatic metastases and $\mathrm{N}$ stage positive status were common factors in the two groups. In addition, transfusion and lymphovascular invasion were risk factors in patients with neoadjuvant treatment (Table 2). However, neoadjuvant treatment related factors (regiments and cycles) did not affect the prognosis.

Table 3 shows the results of multivariate analyses of various parameters evaluated in this study. This revealed only the number of liver metastasis more than three $(\mathrm{HR}=2.051, \mathrm{P}=0.001)$ and $\mathrm{N}$ stage positive $(\mathrm{HR}=1.934$, 
Page 4 of 12

Wu et al. Prediction of recurrence after SCRLM resection

Table 1 Comparison of clinical characteristics between patients with or without neoadjuvant treatment

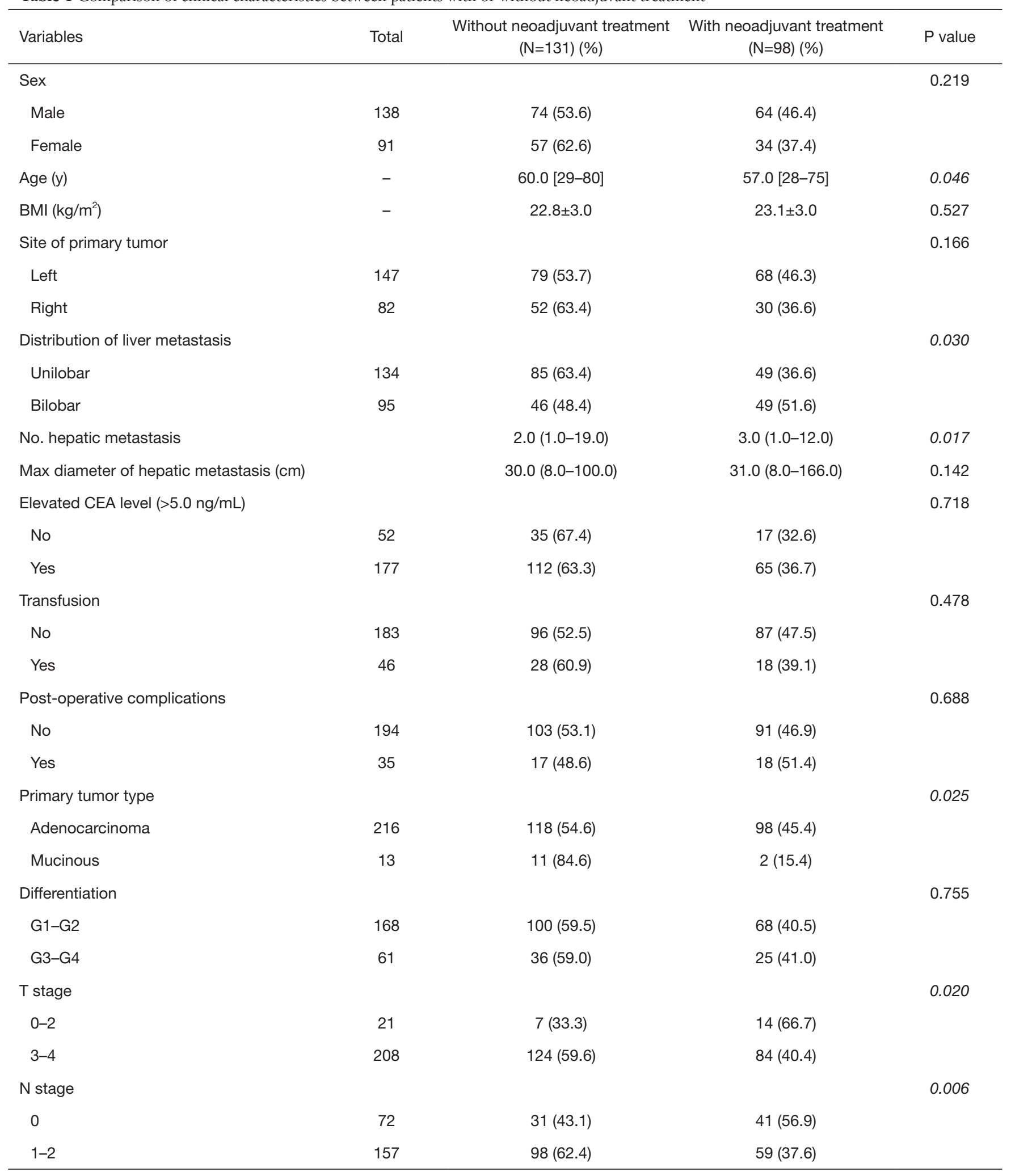

Table 1 (continued) 
Table 1 (continued)

\begin{tabular}{|c|c|c|c|c|}
\hline Variables & Total & $\begin{array}{l}\text { Without neoadjuvant treatment } \\
\qquad(\mathrm{N}=131)(\%)\end{array}$ & $\begin{array}{l}\text { With neoadjuvant treatment } \\
\qquad(\mathrm{N}=98)(\%)\end{array}$ & $P$ value \\
\hline Lymphovascular Invasion & & & & 0.043 \\
\hline No & 115 & $57(49.6)$ & $58(50.4)$ & \\
\hline Yes & 114 & $72(63.2)$ & $42(36.8)$ & \\
\hline Yes & 84 & $52(61.9)$ & $32(38.1)$ & \\
\hline RAS mutation & & & & 0.867 \\
\hline No & 68 & $37(54.4)$ & $31(45.6)$ & \\
\hline Yes & 6 & $5(83.3)$ & $1(16.7)$ & \\
\hline
\end{tabular}

The data are shown as $\mathrm{n}(\%)$ or $\mathrm{n}$ (minimum to maximum). Italic $\mathrm{P}$ values indicate a $\mathrm{P}$ value less than 0.05 .

Table 2 Univariate analysis of prognostic factors

\begin{tabular}{|c|c|c|c|c|c|c|}
\hline Variables & \multicolumn{3}{|c|}{ Without neoadjuvant treatment $(\mathrm{N}=131)$} & \multicolumn{3}{|c|}{ With neoadjuvant treatment $(\mathrm{N}=98)$} \\
\hline Sex & & & 0.957 & & & 0.580 \\
\hline Male & 1 & Ref & & 1 & Ref & \\
\hline Female & 1.014 & $0.616-1.667$ & & 0.858 & $0.498-1.478$ & \\
\hline BMI & 0.993 & $0.913-1.081$ & 0.872 & 1.036 & $0.947-1.132$ & 0.442 \\
\hline Site of primary tumor & & & 0.105 & & & 0.620 \\
\hline Left & 1 & Ref & & 1 & Ref & \\
\hline Right & 1.502 & $0.918-2.456$ & & 0.861 & $0.478-1.553$ & \\
\hline Bilobar & 1.474 & $0.891-2.439$ & & 1.558 & $0.924-2.627$ & \\
\hline No. hepatic metastasis & & & 0.001 & & & 0.117 \\
\hline $1-3$ & 1 & Ref & & 1 & Ref & \\
\hline$>3$ & 2.805 & $1.671-4.709$ & & 1.552 & $0.896-2.687$ & \\
\hline No. hepatic metastasis & & & 0.039 & & & 0.045 \\
\hline 1 & 1 & Ref & & 1 & Ref & \\
\hline$>1$ & 1.705 & $1.028-2.825$ & & 1.598 & $1.010-2.888$ & \\
\hline
\end{tabular}

Table 2 (continued) 
Table 2 (continued)

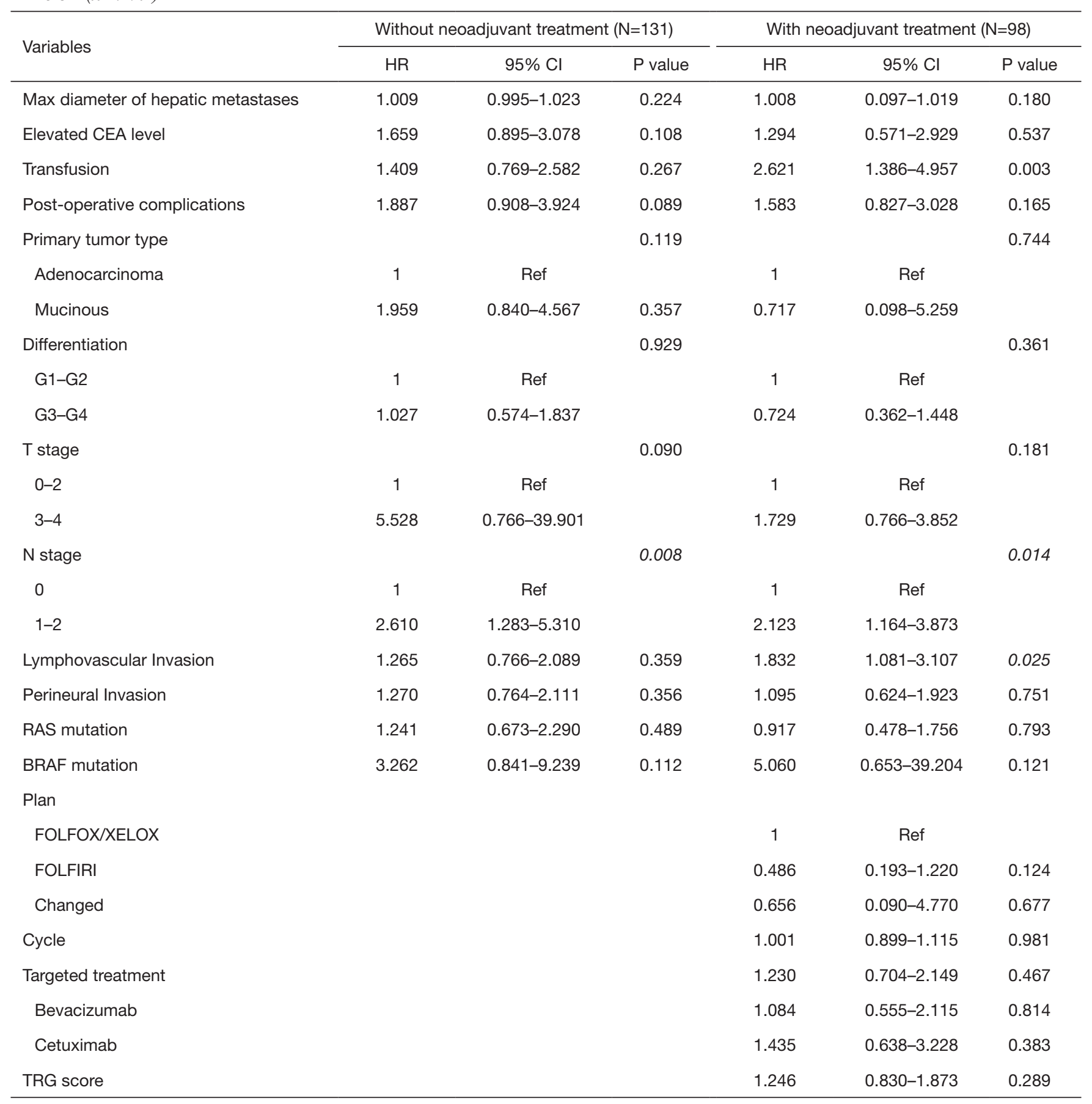

Italic $P$ values indicate a $P$ value less than 0.05 . 
Table 3 Multivariate analysis of prognostic factors

\begin{tabular}{|c|c|c|c|c|c|c|}
\hline Variables & \multicolumn{3}{|c|}{ Without neoadjuvant treatment } & \multicolumn{3}{|c|}{ With neoadjuvant treatment } \\
\hline No. hepatic metastasis $>3$ & 2.051 & $1.398-3.009$ & 0.001 & - & - & - \\
\hline No. hepatic metastasis $>1$ & - & - & - & 1.794 & $1.010-3.419$ & 0.044 \\
\hline Transfusion & - & - & - & 2.327 & $1.221-4.434$ & 0.010 \\
\hline Lymphovascular invasion & - & - & - & 1.636 & $0.913-3.401$ & 0.062 \\
\hline
\end{tabular}

Italic $P$ values indicate a $P$ value less than 0.05 .

Table 4 Risk stratification and analysis

\begin{tabular}{|c|c|c|c|c|c|c|c|c|}
\hline Variables & \multicolumn{4}{|c|}{ Without neoadjuvant treatment } & \multicolumn{4}{|c|}{ With neoadjuvant treatment } \\
\hline Low risk & 27 & 1 & Ref & & 65 & 1 & Ref & \\
\hline Intermediate risk & 83 & 2.714 & $1.211-6.082$ & 0.015 & 25 & 2.111 & $1.180-3.778$ & 0.012 \\
\hline High risk & 21 & 6.478 & $2.694-15.620$ & 0.001 & 8 & 6.668 & $2.736-16.251$ & 0.001 \\
\hline
\end{tabular}

Italic $P$ values indicate a $P$ value less than 0.05 .

$\mathrm{P}=0.004)$ were independent risk factors in patients without neoadjuvant treatment. In those with neoadjuvant treatment, independent risk factors included the number of liver metastasis more than one ( $\mathrm{HR}=1.794, \mathrm{P}=0.044)$, and transfusion during surgery $(\mathrm{HR}=2.327, \mathrm{P}=0.010)$.

\section{Risk factor stratification and comparison with other models}

All independent risk factors were categorized into three groups. A low-risk group comprised patients having no risk factors, and the high-risk group had all risk factors. The stratification showed a significant influence on DFS and compared to the low-risk group, an intermediate-risk group showed a HR more than two. Moreover, HR from the highrisk group could reach more than six in both groups (Table 4).

Three other models have been used to predict recurrence after CRLM resection. To assess the general applicability of these risk scoring systems, we then imported our clinical data to evaluate these models. While our data could fit all inclusion and exclusion criteria of these score systems, in the Zakaria DSS model, only Group 1 and Group 2 could be calculated because we did not routinely resect hepatoduodenal nodes in our patients.

DFS with our risk factors and the other three models are depicted in Figure 1. In patients without neoadjuvant treatment (Figure 1A), all models could stratify patients into different DFS levels. However, in patients with neoadjuvant treatment (Figure 1B), only our model and the Fong CRS score could discriminate patients and overlap of DFS could be found in the Konopke score. In addition, in the Zakaira model, only a few Group 1 patients reached a significant difference compared with Group 2 patients.

The C-index in our models was also calculated (Table 5) and showed that although our present model performed better in discriminating patients in different risks of recurrence than the other three models, the C-index could only reach around 0.71 . Moreover, the other three models showed low ability in recurrence time prediction $(\mathrm{C}$-index no more than 0.60 ).

The survival curves of all patients in the validation group are presented in Figure 2 and show that recurrence within two years of follow-up was present in 94 patients (73\%), of which 66 had neoadjuvant therapy. After applying our score system, the survival curves were also significantly classified (without neoadjuvant therapy, $\mathrm{P}=0.002$; with neoadjuvant therapy, $\mathrm{P}=0.046)$.

\section{Discussion}

The surgical benefit of simultaneous resection, including 

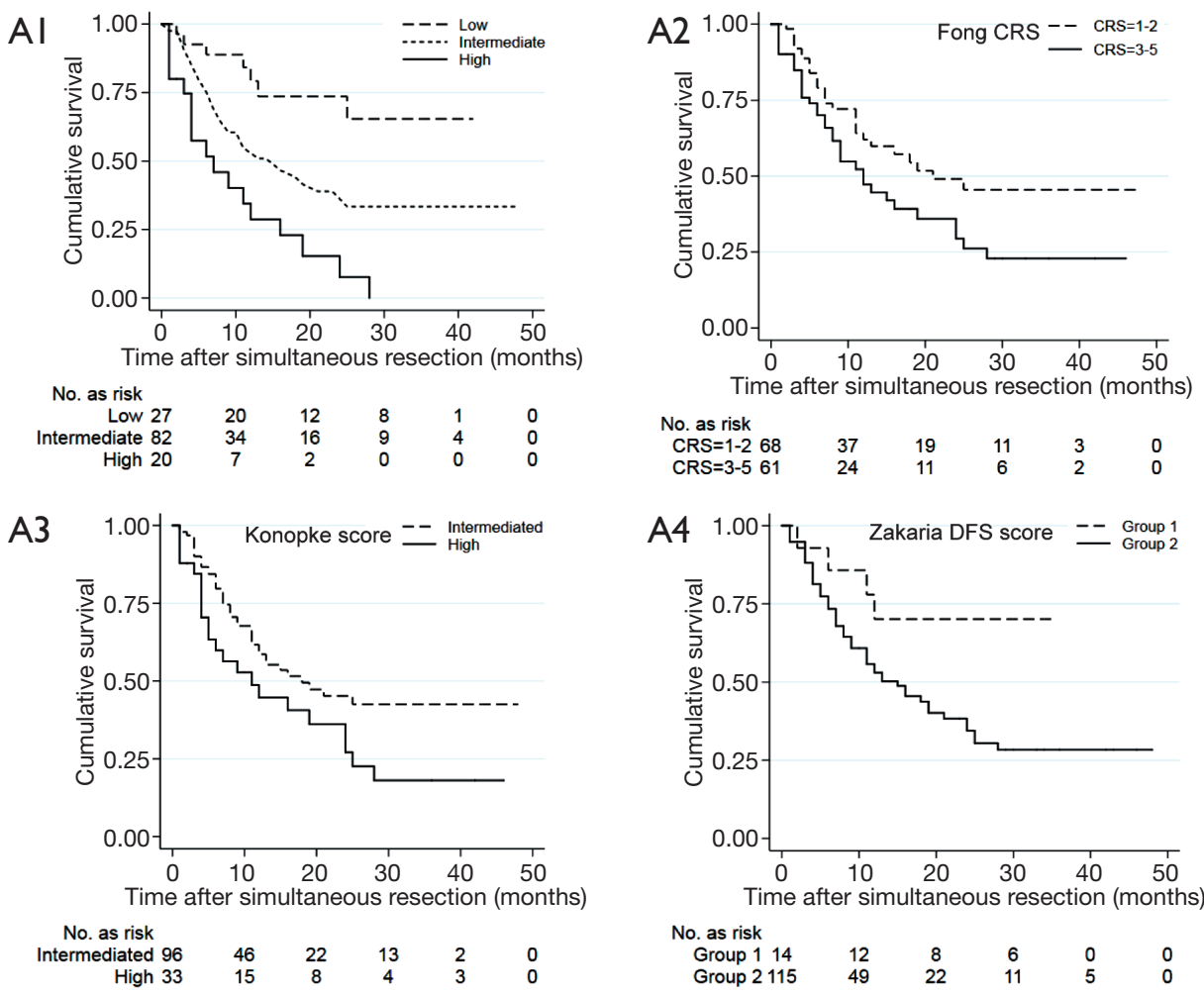

B I
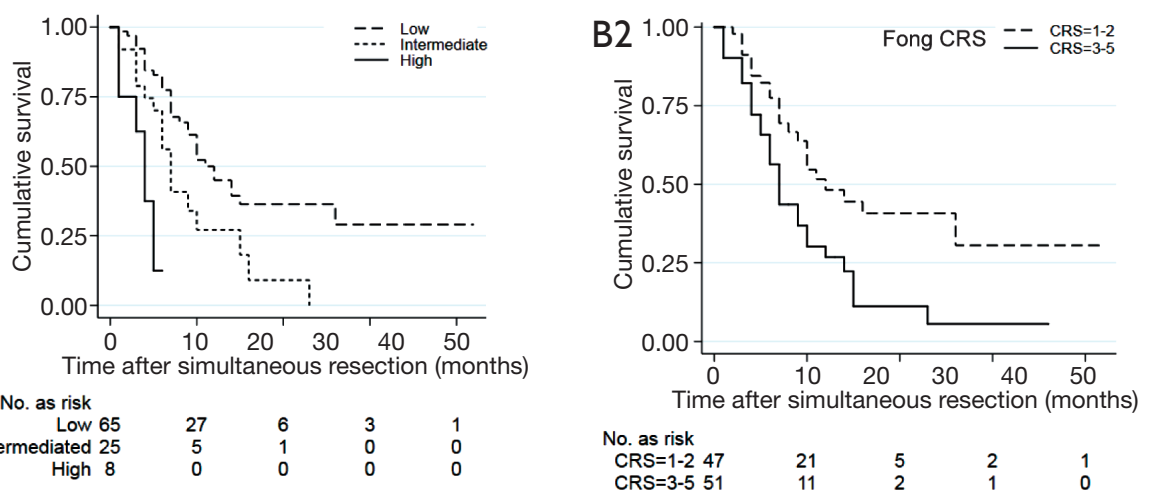

B3
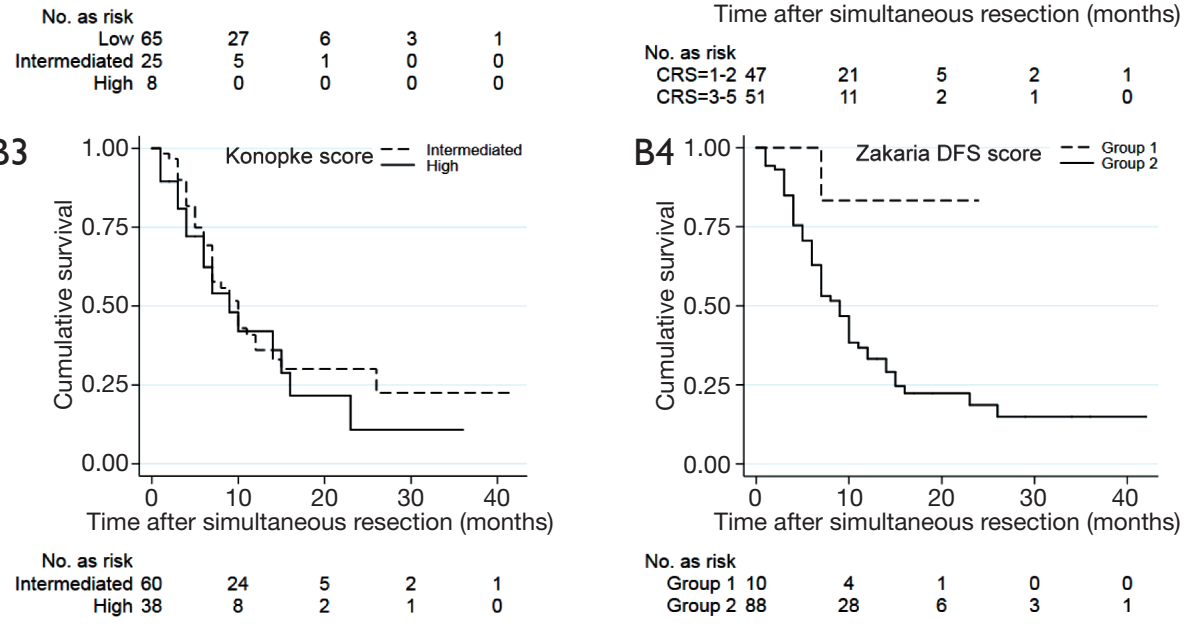

Figure 1 Risk scoring system to predict recurrence after simultaneous resection of SCRLM in patients without (A) and with (B) neoadjuvant treatment. (A1,B1) Our scoring system; (A2,B2) Fong CRS; (A3,B3) Konopke, and (A4,B4) Zakaria DFS score. SCRLM, synchronous colorectal cancer liver metastasis; CRS, clinical recurrence score; DFS, disease-free survival. 
less operation time, length of hospitalization and cost, has been widely accepted. Controversy of prognosis still exists around the simultaneous resection of SCRLM and neoadjuvant treatment. Based on data from the LiverMetSurvey, Adam showed that patients with low rectal primary tumors needing radiotherapy, complex tumors, or major hepatectomy ( $\geq 3$ segments) did not benefit from simultaneous resection and had a worse 5-year survival (40\%) (19). Conversely, Mayo and Silberhumer found that simultaneous resection was associated with similar long-term cancer outcomes compared with staged procedures, although major resection was more common in the staged group $(7,9)$. It is also clear that patients with initially unresectable CRLM can benefit from a combination of neoadjuvant treatment and radical surgery, and even those with resectable CRLM obtained a benefit in PFS from neoadjuvant treatment in the 40983 Clinical Trial (20) especially when they had elevated CEA and stable performance status (21), although few researchers have obtained opposite results (22). However, given the increasing number of patients undergoing neoadjuvant treatment and simultaneous resection, there is an urgent

Table 5 Concordance probability estimates of progression-free survival $(95 \% \mathrm{CI})$

\begin{tabular}{lcc}
\hline Model & $\begin{array}{c}\text { Without neoadjuvant } \\
\text { treatment }\end{array}$ & $\begin{array}{c}\text { With neoadjuvant } \\
\text { treatment }\end{array}$ \\
\hline Present & $0.71(0.66-0.75)$ & $0.71(0.67-0.73)$ \\
Fong CRS & $0.58(0.52-0.62)$ & $0.60(0.52-0.68)$ \\
Konopke score & $0.57(0.51-0.63)$ & $0.51(0.43-0.58)$ \\
Zakaria DFS score & $0.54(0.49-0.59)$ & $0.55(0.51-0.61)$ \\
\hline
\end{tabular}

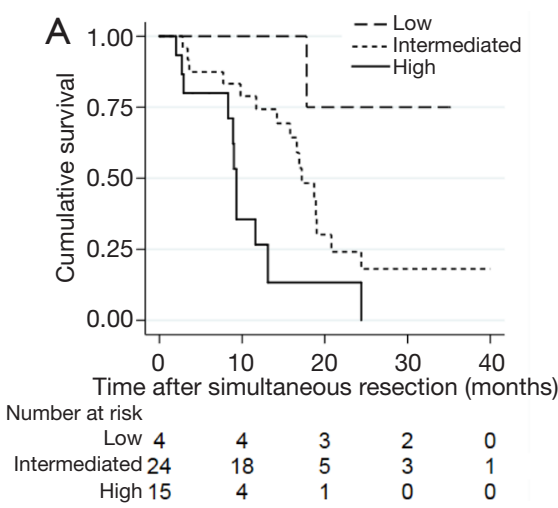

need to study prognostic factors in different clinical situations. In this study, we investigated the risk factors for recurrence in patients with SCRLM after simultaneous resection. More importantly, once neoadjuvant treatment was considered as a stratification factor, different prognosis models were presented. While we also validated other published predictive models in our data, the predictive values were unsatisfactory.

This may be explained by the innate difference in patients included in most studies and score systems. The most widely used system to predict recurrence after resection of CRLM is the CRS system, which was established by Fong in 1999 based on 1,001 cases. However, liver metastasis are mostly metachronous, and factors concerning neoadjuvant treatment were not separately analyzed in the CRS system or others investigating recurrence, including those established by Konopke and Zakaria. Moreover, certain factors, such as hepatoduodenal node resection were not adaptable to all centers. However, external validation has addressed these problems. In a study by Reissfelder (23), validating the performance of different score systems in predicting disease-specific survival proved that only the MSKCC, Fong CRS), and Iwatsuki score produced reliable results (24). Further, Roberts compared almost all clinical scores to detect their value in predicting 10-years survival and found no score system reached sufficient discriminatory accuracy (25). When factors such as neoadjuvant treatment were included, Wimmer found that all score systems except the Konopke score were predictive for DFS and OS (26), which was an improvement on our results. Overall, the diverse conclusions from different centers may not only reflect differences in treatment strategies and time, but also the inclusion of patients of different status and risk factors

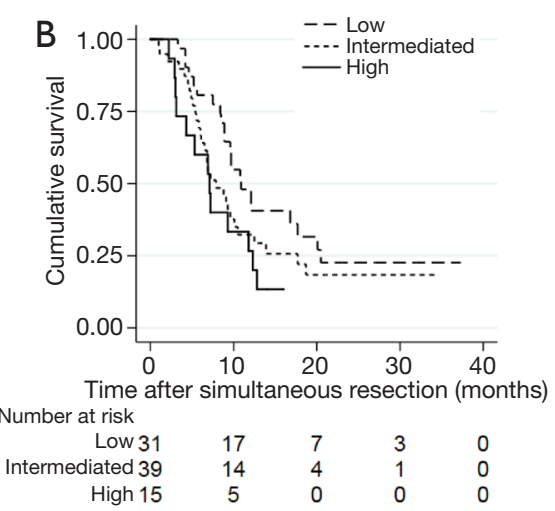

Figure 2 Applying risk score system in validation group. (A) Patients without neoadjuvant treatment; (B) patients with neoadjuvant treatment. 
including differences in race and other factors.

Nonetheless, most studies confirm that factors including the status and number of liver metastasis and lymph node infiltration are important as shown in in the Fong CRS and Zakaria scores, and confirmed in our results in patients not receiving neoadjuvant treatment. The numbers of liver metastasis undoubtedly increases the probability of recurrence, especially liver recurrence after surgery, and micro-metastasis, which cannot be detected by images and IOUs, always correlate with large numbers of detectable CRLM. Moreover, lymph node involvement indicates the local dissemination of tumors and has been widely recognized as a risk factor in all stages of CRC. However, risk factors in patients receiving neoadjuvant treatment differ. While the response to treatment in pathological features such as TRG and $\mathrm{N}$ stage might determine the prognosis, in our data, TRG and $\mathrm{N}$ stage did not predict the prognosis. This may be because of the different regression mode among primary tumors, micro-metastasis, and positive lymph nodes, or may reflect the limited numbers in our study. The response from liver metastasis was also hard to define. Although there are some studies establishing TRG scores with liver lesions (26), generally this association has not been made. Transfusion also affects the survival of patients after neoadjuvant treatment, as seen in the study by Zakaria and others (27). Partially different from patients not receiving radiochemotherapy, transfusion in those with neoadjuvant treatment indicated not only the severity of disease, but also a decreased body reserve and adaptability. In extreme conditions, chemotherapy must be reduced or even stopped because of severe toxicity which attenuates the anti-tumor effect of drugs. Notably, we observed two cut-offs in the numbers of liver metastasis in different situations, which has also been observed in other research, and these were mainly based on statistical calculation and indicate unstable evaluation dimensions for liver metastasis. While Sasaki established a "Tumor Burden Score" by systematically integrating radiographic or pathological features for risk stratification, the score requires further external validation (28). Overall, a comprehensive evaluation combining position, number, size, local invasive and other factors through imaging, pathology, and even radiomics (29) should be established and evaluated.

The C-index we demonstrated was not sufficient to discriminate patients for their DFS, although our models were better than others and served the purpose of an external validation of others as we used more detailed inclusion and category criteria. More importantly, the failure of our models to be accurate means that further investigation is required to determine the presence and role of hitherto unidentified factors. This will require increasing the cohort size and evaluating a broader range of factors as well as external validation. A combination of other factors, rather than clinical features, such as liquid biopsy and radiomics should also be considered (30).

The present study had several limitations. Firstly, the case numbers were small and more factors concerning reoccurrence and prognosis need to be considered. The effect of drugs should also be intensively studied, especially in cycles and targeted therapy. Moreover, we did not evaluate the prognostic impact of BRAF or RAS mutation. Not all patients in our center underwent gene mutation examination, possibly because of the cost of this, or for other personal reasons. In any case, the mutation rate of BRAF in Asians is comparably lower than in other groups (31), and its real impact requires broader validation. Furthermore, overall survival was not further evaluated because of the limited follow-up time, and future studies are in progress.

Benefiting from modern surgical technologies and care, more and more patients with synchronous CRLM are undergoing simultaneous resection combined with neoadjuvant treatment, and a predictive model is essential to optimize treatment decisions and supervision. Although current scoring systems to clarify prognosis are unreliable, certain risk factors have been identified. The future development of a risk score system, especially for patients with neoadjuvant treatment, requires studies including more cases and the evaluation of a broader range of risk factors.

\section{Acknowledgments}

Funding: This work was partially supported by the Science and Technology Commission of Shanghai Municipality (19511121202, 20DZ1100101).

\section{Footnote}

Reporting Checklist: The authors have completed the TRIPOD reporting checklist. Available at https://dx.doi. org/10.21037/atm-21-2595

Data Sharing Statement: Available at https://dx.doi. org/10.21037/atm-21-2595

Conflicts of Interest: All authors have completed the ICMJE uniform disclosure form (available at https://dx.doi. 
org/10.21037/atm-21-2595). The authors have no conflicts of interest to declare.

Ethical Statement: The authors are accountable for all aspects of the work in ensuring that questions related to the accuracy or integrity of any part of the work are appropriately investigated and resolved. The study was conducted in accordance with the Declaration of Helsinki (as revised in 2013) and was approved by the Medical Ethics Committee of Fudan University Shanghai Cancer Center. Written informed consent was obtained from all participants.

Open Access Statement: This is an Open Access article distributed in accordance with the Creative Commons Attribution-NonCommercial-NoDerivs 4.0 International License (CC BY-NC-ND 4.0), which permits the noncommercial replication and distribution of the article with the strict proviso that no changes or edits are made and the original work is properly cited (including links to both the formal publication through the relevant DOI and the license). See: https://creativecommons.org/licenses/by-nc-nd/4.0/.

\section{References}

1. Siegel RL, Miller KD, Fedewa SA, et al. Colorectal cancer statistics, 2017. CA Cancer J Clin 2017;67:177-93.

2. Chen $W$, Zheng R, Baade PD, et al. Cancer statistics in China, 2015. CA Cancer J Clin 2016;66:115-32.

3. Leporrier J, Maurel J, Chiche L, et al. A populationbased study of the incidence, management and prognosis of hepatic metastases from colorectal cancer. Br J Surg 2006;93:465-74.

4. Oliphant R, Nicholson GA, Horgan PG, et al. Contribution of surgical specialization to improved colorectal cancer survival. Br J Surg 2013;100:1388-95.

5. Kassahun WT. Unresolved issues and controversies surrounding the management of colorectal cancer liver metastasis. World J Surg Oncol 2015;13:61.

6. Mentha G, Majno PE, Andres A, et al. Neoadjuvant chemotherapy and resection of advanced synchronous liver metastases before treatment of the colorectal primary. Br J Surg 2006;93:872-8.

7. Mayo SC, Pulitano C, Marques H, et al. Surgical management of patients with synchronous colorectal liver metastasis: a multicenter international analysis. J Am Coll Surg 2013;216:707-16; discussion 716-8.

8. Slesser AA, Chand M, Goldin R, et al. Outcomes of simultaneous resections for patients with synchronous colorectal liver metastases. Eur J Surg Oncol 2013;39:1384-93.

9. Silberhumer GR, Paty PB, Denton B, et al. Longterm oncologic outcomes for simultaneous resection of synchronous metastatic liver and primary colorectal cancer. Surgery 2016;160:67-73.

10. Li Y, Bi X, Zhao J, et al. Simultaneous hepatic resection benefits patients with synchronous colorectal cancer liver metastases. Chin J Cancer Res 2016;28:528-35.

11. Abelson JS, Michelassi F, Sun T, et al. Simultaneous Resection for Synchronous Colorectal Liver Metastasis: the New Standard of Care? J Gastrointest Surg 2017;21:975-82.

12. Reddy SK, Pawlik TM, Zorzi D, et al. Simultaneous resections of colorectal cancer and synchronous liver metastases: a multi-institutional analysis. Ann Surg Oncol 2007;14:3481-91.

13. Luo Y, Wang L, Chen C, et al. Simultaneous liver and colorectal resections are safe for synchronous colorectal liver metastases. J Gastrointest Surg 2010;14:1974-80.

14. Wu Y, Liu F, Song W, et al. Safety evaluation of simultaneous resection of colorectal primary tumor and liver metastasis after neoadjuvant therapy: A propensity score matching analysis. Am J Surg 2019;218:894-8.

15. Lee WS, Kim MJ, Yun SH, et al. Risk factor stratification after simultaneous liver and colorectal resection for synchronous colorectal metastasis. Langenbecks Arch Surg 2008;393:13-9.

16. Fong Y, Fortner J, Sun RL, et al. Clinical score for predicting recurrence after hepatic resection for metastatic colorectal cancer: analysis of 1001 consecutive cases. Ann Surg 1999;230:309-18; discussion 318-21.

17. Konopke R, Kersting S, Distler M, et al. Prognostic factors and evaluation of a clinical score for predicting survival after resection of colorectal liver metastases. Liver Int 2009;29:89-102.

18. Zakaria S, Donohue JH, Que FG, et al. Hepatic resection for colorectal metastases: value for risk scoring systems? Ann Surg 2007;246:183-91.

19. Adam R, de Gramont A, Figueras J, et al. Managing synchronous liver metastases from colorectal cancer: a multidisciplinary international consensus. Cancer Treat Rev 2015;41:729-41.

20. Nordlinger B, Sorbye H, Glimelius B, et al. Perioperative FOLFOX4 chemotherapy and surgery versus surgery alone for resectable liver metastases from colorectal cancer 
(EORTC 40983): long-term results of a randomised, controlled, phase 3 trial. Lancet Oncol 2013;14:1208-15.

21. Sorbye H, Mauer M, Gruenberger T, et al. Predictive factors for the benefit of perioperative FOLFOX for resectable liver metastasis in colorectal cancer patients (EORTC Intergroup Trial 40983). Ann Surg 2012;255:534-9.

22. de Ridder JAM, van der Stok EP, Mekenkamp LJ, et al. Management of liver metastases in colorectal cancer patients: A retrospective case-control study of systemic therapy versus liver resection. Eur J Cancer 2016;59:13-21.

23. Reissfelder C, Rahbari NN, Koch M, et al. Validation of prognostic scoring systems for patients undergoing resection of colorectal cancer liver metastases. Ann Surg Oncol 2009;16:3279-88.

24. Iwatsuki S, Dvorchik I, Madariaga JR, et al. Hepatic resection for metastatic colorectal adenocarcinoma: a proposal of a prognostic scoring system. J Am Coll Surg 1999;189:291-9.

25. Roberts KJ, White A, Cockbain A, et al. Performance of prognostic scores in predicting long-term outcome following resection of colorectal liver metastases. Br J Surg
2014;101:856-66.

26. Wimmer K, Schwarz C, Szabo C, et al. Impact of Neoadjuvant Chemotherapy on Clinical Risk Scores and Survival in Patients with Colorectal Liver Metastases. Ann Surg Oncol 2017;24:236-43.

27. Zakaria S, Donohue JH, Que FG, et al. Hepatic resection for colorectal metastases: value for risk scoring systems? Ann Surg 2007;246:183-91.

28. Sasaki K, Margonis GA, Andreatos N, et al. The prognostic utility of the "Tumor Burden Score" based on preoperative radiographic features of colorectal liver metastases. J Surg Oncol 2017;116:515-23.

29. Lambin P, Leijenaar RTH, Deist TM, et al. Radiomics: the bridge between medical imaging and personalized medicine. Nat Rev Clin Oncol 2017;14:749-62.

30. Jones RP, Brudvik KW, Franklin JM, et al. Precision surgery for colorectal liver metastases: Opportunities and challenges of omics-based decision making. Eur J Surg Oncol 2017;43:875-83.

31. Yokota T, Ura T, Shibata N, et al. BRAF mutation is a powerful prognostic factor in advanced and recurrent colorectal cancer. Br J Cancer 2011;104:856-62.
Cite this article as: Wu Y, Guo T, Xu Z, Liu F, Cai S, Wang L, $\mathrm{Xu}$ Y. Risk scoring system for recurrence after simultaneous resection of colorectal cancer liver metastasis. Ann Transl Med 2021;9(12):966. doi: 10.21037/atm-21-2595 


\section{Supplementary}

A
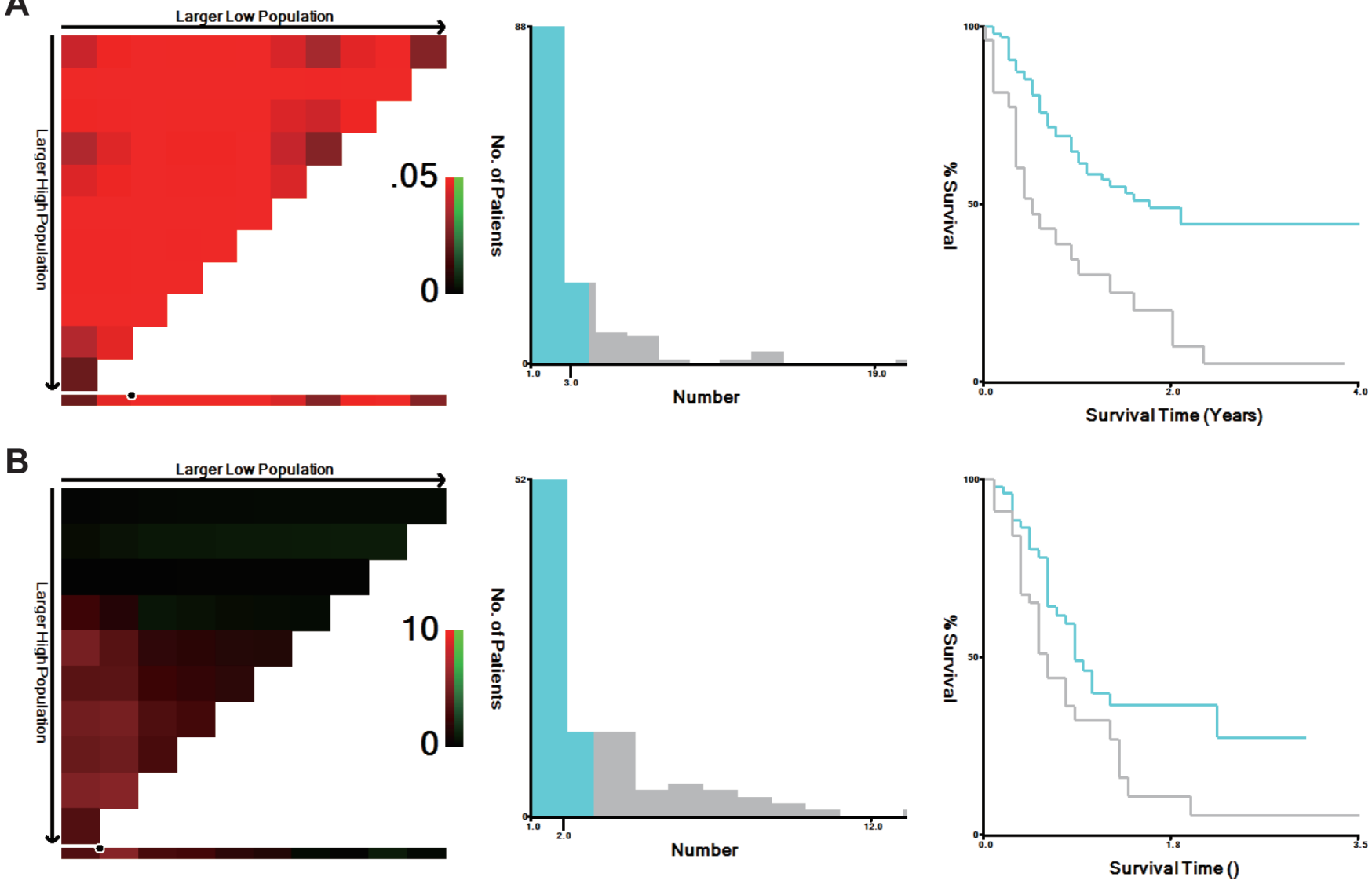

Figure S1 Classified survival according to the number of liver metastasis, using X-tile, in patients without (A) and with (B) neoadjuvant treatment. 


\begin{tabular}{lc}
\hline Variables & Total \\
\hline Sex & \\
Male & 121 \\
Female & 78 \\
Age $(\mathrm{y})$ & 57.8 \\
$\mathrm{BMI}\left(\mathrm{kg} / \mathrm{m}^{2}\right)$ & 22.9
\end{tabular}

Site of primary tumor

Left

Right

Distribution of liver metastasis

Unilobar

Bilobar

No. hepatic metastasis

Max diameter of hepatic metastasis $(\mathrm{cm})$

Elevated CEA level (>5.0 ng/mL)

$\begin{array}{lr}\text { No } & 38 \\ \text { Yes } & 161 \\ \text { Neoadjuvant therapy } & 120\end{array}$

Neoadjuvant therapy

Transfusion

No

Yes

Post-operative complications

No

Yes

Primary tumor type

Adenocarcinoma

Mucinous

Differentiation

G1-G2

\section{T stage}

0-2

30

3-4

$\mathrm{N}$ stage

0

Lymphovascular invasion

Perineural Invasion

RAS mutation

No

71

Yes

80

BRAF mutation 